\title{
Immunization by Replication-Competent, Controlled Viral Pathogen - A Novel Approach Worth Exploring for Diseases Refractory to Effective Conventional Vaccination?
}

\author{
Richard Voellmy ${ }^{1,2^{*}}$, David C Bloom ${ }^{3}$, Nuria Vilaboa ${ }^{4,5}$ \\ ${ }^{1}$ Department of Physiological Sciences, University of Florida College of Veterinary Sciences, Gainesville, FL, USA \\ ${ }^{2}$ HSF Pharmaceuticals SA, La Tour-de-Peilz, Switzerland \\ ${ }^{3}$ Department of Molecular Genetics \& Microbiology, University of Florida College of Medicine, Gainesville, FL, USA \\ ${ }^{4}$ Hospital Universitario La Paz-IdiPAZ, Madrid, Spain \\ ${ }^{5}$ CIBER de Bioingenieria, Biomateriales y Nanomedicina, CIBER-BBN, Spain \\ "Corresponding author: Richard Voellmy, HSF Pharmaceuticals SA, Av. de Sully 67, 1814 La Tour-de-Peilz, Switzerland, Tel: 215340260; E-mail: \\ rvoellmy@hsfpharma.com
}

Received date: May 28, 2015; Accepted date: June 27, 2015; Published date: June 30, 2015

Copyright: (c) 2015 Richard Voellmy, et al. This is an open-access article distributed under the terms of the Creative Commons Attribution License, which permits unrestricted use, distribution, and reproduction in any medium, provided the original author and source are credited.

Keywords: Controlled replication; Gene switch; Ligand-dependent transactivator; Heat shock; Immunization; Vaccination; Virus; Pathogen; Conditional replication

\section{Description}

Edward Jenner tested the first vaccine that was aimed at small pox in 1796. Overall, vaccination has been a very successful approach for disease prevention. However, more than two centuries after the first vaccine use, there still remain diseases and conditions that cannot be effectively prevented or treated by vaccination. Unfortunately, the diseases that have remained refractory to vaccination or that are insufficiently protected against by vaccination include diseases of global concern including influenza/flu, tuberculosis, malaria, HIV/ AIDS, and oral and genital herpes. While vaccine development efforts based on the "classical" paradigm (described below) are continuing, it may be appropriate to also consider novel approaches that may have a potential for inducing qualitatively and/or quantitatively superior immune responses.

Most currently licensed vaccines are either subunit vaccines (comprising isolated proteins or protein fractions) or attenuated forms of disease-causing microorganisms. Attenuation of pathogens is achieved by their killing or by genetic disablement of their replicative ability. While attenuation provides the required safety, it almost universally compromises the ability of so-modified pathogens to induce robust inflammatory responses, which translates in suboptimal humoral and T-cell responses [1-4]. It is obviously not feasible to compare immune responses elicited by a wild type pathogenic agent vs. those induced by an attenuated agent. However, several studies have compared immune responses elicited by viral vectors that were attenuated but had retained residual replicative ability and corresponding vectors that were replication-incompetent [5-8]. Results indicated that attenuated viruses that retained some replication ability induced more complete and more potent immune responses than non-replicating comparison viruses.

Based on these considerations and findings, we hypothesized that a genetically altered viral pathogen that can be transiently activated in an inoculation site region to (locally) replicate with an efficiency approaching that of the corresponding wild type agent would be a superior immunization agent to a conventional vaccine [9]. Replication is understood here as propagation of the agent.
How could one confine replication of a viral pathogen to the inoculation site region as well as limit the duration of its replication? It is well known that viruses will not remain in the region in which they have been administered but will disseminate within the host organism. Therefore, a suitable control mechanism will need to be capable of activating replication in the inoculation site region but not elsewhere. It is believed that such a control mechanism will need to respond to a physical "signal" that can be targeted to the inoculation site region. One possibility, representing perhaps the only practicable solution available at this time, will be the use of a highly heat-inducible heat shock protein gene (HSP) promoter for controlling the expression of replication-essential genes of a pathogenic virus. Certain HSP promoters such as the human HSP70B promoter have very low basal activity that can be induced several thousand fold by heat activation $[10,11]$. The promoters appear to be capable of being activated in all mammalian cell types. Activation of the HSP70B promoter requires a heat dose that is essentially outside of the physiological range but can be readily tolerated by a human subject $\left(44-45^{\circ} \mathrm{C}\right.$ for $\left.5-10 \mathrm{~min}\right)$. Should this be desired, it would be possible to modify the promoter so that it responds to a lower heat dose [12]. Heat can be targeted in many ways. In the case of intradermal or subcutaneous inoculation, a simple and robust approach would involve the application of a heating patch. Such a patch may be manufactured using well-known and inexpensive technology (of the kind used in commercial products such as ThermaCare).

The most critical issue with an immunization agent that can be activated to replicate with near wild type efficiency will be safety from inadvertent transient activation or, worse, run-away activation. A practical (not safety-related) question that also needs to be addressed arises from the mode of regulation of HSP promoters. Exposure of a cell to heat (even sustained heat) results in a transient activation of heat shock transcription factor 1 (HSF1) that then binds to and mediates transcription from HSP promoters. HSF1 reverts to an inactive form within at most a few hours from heat activation. As a consequence, HSP promoters per se are not well adapted for controlling genes of a pathogen whose activity is required during much of the replication cycle or that need to be active at different times in the cycle. We suggested that adequate safety from inadvertent activation, whether caused by exceptional circumstances (e.g., ischemia, intoxication, etc.) or a recombination event, could come from using a dual-responsive gene switch for controlling at least two replication-essential genes of a viral pathogen that is to be used as an 
Citation: Richard Voellmy, David C Bloom, Nuria Vilaboa (2015) Immunization by Replication-Competent, Controlled Viral Pathogen - A Novel Approach Worth Exploring for Diseases Refractory to Effective Conventional Vaccination?. J Vaccines Vaccin 6: 286. doi: $10.4172 / 2157-7560.1000286$

Page 2 of 3

immunization agent [9]. Dual-responsive gene switches that are activated by a combination of heat and a small-molecule regulator (SMR) were described previously and are known to stringently control expression of target genes both in vitro and in vivo [13-15]. The gene switches consist of (i) an SMR-activated transactivator that is expressed from a promoter cassette which is activated both by heat and the transactivator, and (ii) a transactivator-responsive promoter for driving a gene of interest. A viral pathogen that has two replication-essential genes controlled by a dual-responsive gene switch is represented in Figure 1A, and how the gene switch operates is illustrated in Figure 1B. Upon administration of such a replicationcompetent controlled pathogen to a chosen inoculation region, replication of the agent is triggered by a localized heat treatment, e.g., application of a heating patch, in the presence of SMR. The SMR may be provided systemically or may be co-administered with the immunizing agent. The activated gene switch in the infected cells will remain active until clearance of the SMR has occurred or the cells have been lysed by the replicating agent. Thereafter, the agent is safely disabled. For as long as the agent persists in the inoculation region, deliberate re-activation of replication will be possible.
A

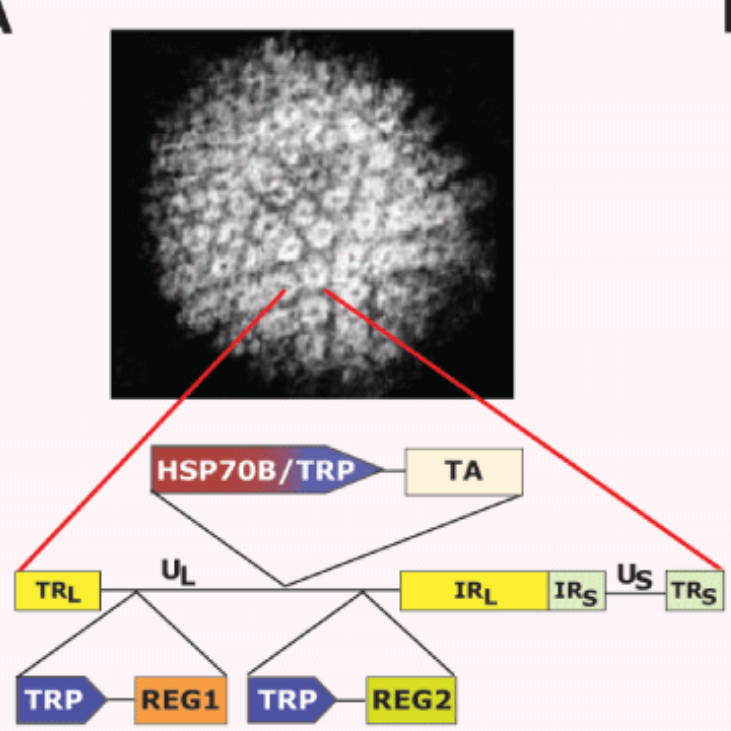

B

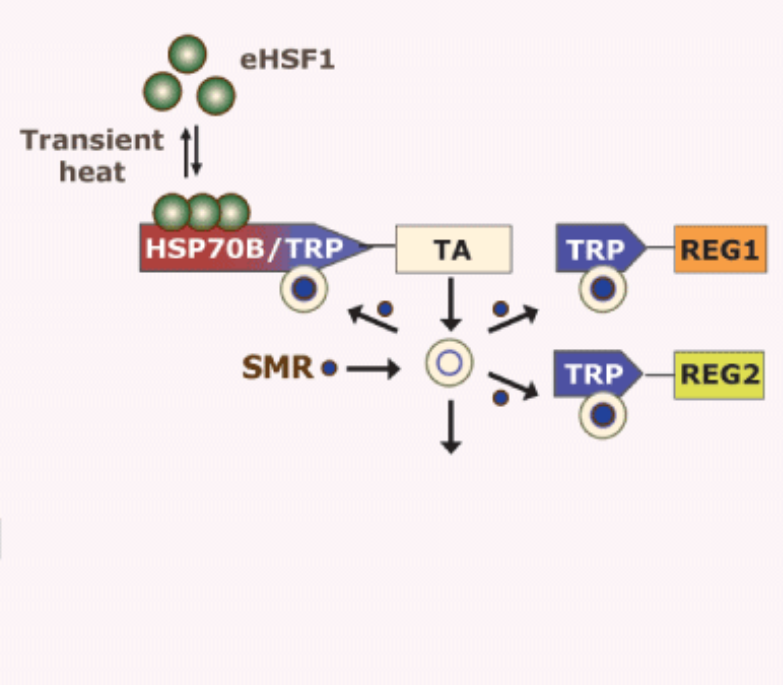

Figure 1: A. Replication-competent controlled HSV. An HSV genome is represented schematically in which replication-essential genes REG1 and REG2 were placed under control of a transactivator-responsive promoter (TRP) and a transactivator gene (TA) controlled by a promoter cassette (HSP70B/TRP) comprising an HSP70B and a TRP promoter was inserted. $\mathrm{TR}_{\mathrm{L}}, \mathrm{TR}_{\mathrm{S}}$ : long and short terminal repeats; $\mathrm{U}_{\mathrm{L}}$, $\mathrm{U}_{\mathrm{S}}$ : long and short unique regions; $\mathrm{IR}_{\mathrm{L}}$, IR $\mathrm{IR}_{\mathrm{S}}$ long and short internal repeats. B. Illustration of an activated dual-responsive gene switch. eHSF1: host cell HSF1; SMR: small-molecule regulator. The extra arrow beginning at the transactivator symbol (circle in a circle) indicates elimination/ dilution of transactivator.

Is it feasible to construct such replication-competent controlled viruses? Will they have a potential for inducing better immune responses than conventional vaccines? We placed two replicationessential genes of a wild type strain of Herpes Simplex Virus 1 (HSV-1) under the control of a dual-responsive gene switch (manuscript in preparation). The transactivator integrated in the latter gene switch was activated by antiprogestin mifepristone and highly related compounds (but not progestins). In vitro, the recombinant virus replicated essentially as efficiently as the wild type virus after heat activation in the presence of antiprogestin, but not in the absence of either antiprogestin or heat treatment. Replication of the virus was also stringently controlled in the mouse. In immunization/challenge experiments, once activated recombinant virus was significantly more protective than a corresponding non-replicating virus. These findings are encouraging and warrant an exploration of the full potential of the proposed immunization approach.

How broadly applicable may this technology be? Replication of a viral pathogen can only be controlled by the above-described dualresponsive gene switch if the pathogen normally engages the host transcriptional machinery for expression of its genes. This limitation is less important than one might suspect at first sight (Table 1). There is a long history of use of (replication-defective and attenuated) viral vectors for expressing heterologous antigens $[16,17]$. A replicationcompetent controlled virus may serve as a potent immunization platform. For the reasons that were discussed at the beginning of this communication, expression of heterologous antigens in the context of vigorous viral replication is expected to result in superior immune responses. Furthermore, viral pathogens that do not utilize the host transcriptional machinery may be regulated through complementation. A replication-essential gene of such a virus may be disabled and the gene product provided by a co-infecting (replicationdisabled or replication-competent controlled) virus that expresses the replication-essential gene under the control of a dual-responsive gene switch. It is noted that whenever viral vectors are utilized as immunization or oncolytic agents, the question arises whether preexisting immunity will substantially reduce the efficacy of these agents. Many studies have addressed this question for HSV, our preferred backbone for controlled virus construction (cited in ref.9). The prevailing answer is that pre-existing immunity has little effect or only 
Citation: Richard Voellmy, David C Bloom, Nuria Vilaboa (2015) Immunization by Replication-Competent, Controlled Viral Pathogen - A Novel Approach Worth Exploring for Diseases Refractory to Effective Conventional Vaccination?. J Vaccines Vaccin 6: 286. doi: $10.4172 / 2157-7560.1000286$

Page 3 of 3

relatively minor effects on immune responses to HSV-delivered antigens or on anti-tumour efficacy of oncolytic HSV.

\begin{tabular}{|l|l|}
\hline Agent & Potential use \\
\hline Replication-competent controlled HSV* & Preventative or therapeutic immunization against HSV \\
\cline { 2 - 3 } & Oncolytic therapy \\
\hline $\begin{array}{l}\text { Replication-competent controlled HSV* expressing heterologous antigens (e.g., } \\
\text { from HIV, influenza, M. tuberculosis) }\end{array}$ & Immunization against HIV/AIDS, flu, tuberculosis, etc. \\
\hline $\begin{array}{l}\text { Replication-competent controlled HSV*+co-infecting viral pathogen having at least } \\
\text { one replication-essential gene controlled by the dual-responsive gene switch } \\
\text { (present in the controlled HSV) }\end{array}$ & $\begin{array}{l}\text { Immunization against the co-infecting viral pathogen (e.g., adenovirus, papilloma, } \\
\text { polyoma, otherpesvirus, etc.) }\end{array}$ \\
\hline $\begin{array}{l}\text { Replication-competent controlled HSV* expressing a replication-essential gene of } \\
\text { a co-infecting viral pathogen under gene switch control+co-infecting viral pathogen } \\
\text { with disabled replication-essential gene }\end{array}$ & $\begin{array}{l}\text { Immunization against the co-infecting viral pathogen capable of co-infection) } \\
\text { pathentially any viral }\end{array}$ \\
\hline
\end{tabular}

Table 1: Potential applications for a replication-competent controlled HSV. ${ }^{*}$ The controlled HSV may express heterologous proteins that enhance immune functions (e.g., proteins functioning as cytokines). In addition, or in the alternative, certain immune evasion mechanisms may be disabled (e.g., ICP47).

\section{Conflict of interest}

Richard Voellmy is the founder and CEO of HSF Pharmaceuticals SA, which company has sponsored research relating to conditionally replicating viruses in the laboratories of the co-authors. The coauthors report no independent conflict of interest.

\section{References}

1. Pulendran B, Ahmed R (2006) Translating innate immunity into immunological memory: implications for vaccine development. Cell 124: 849-863.

2. Mina MJ, Klugman KP (2013) Pathogen replication, host inflammation, and disease in the upper respiratory tract. Infect Immun 81: 625-628.

3. Marois I, Cloutier A, Garneau E, Richter MV (2012) Initial infectious dose dictates the innate, adaptive, and memory responses to influenza in the respiratory tract. J Leukoc Biol 92: 107-121.

4. Boonnak K, Paskel M, Matsuoka Y, Vogel L, Subbarao K (2012) Evaluation of replication, immunogenicity and protective efficacy of a live attenuated cold-adapted pandemic H1N1 influenza virus vaccine in non-human primates. Vaccine 30: 5603-5610.

5. Peng B, Wang LR, Gomez-Roman VR, Davis-Warren A, Montefiori DC, et al. (2005) Replicating rather than nonreplicating adenovirus-human immunodeficiency virus recombinant vaccines are better at eliciting potent cellular immunity and priming high-titer antibodies. J Virol 79: 10200-10209.

6. Halford WP, Weisend C, Grace J, Soboleski M, Carr DJ, et al. (2006) ICP0 antagonizes Stat I-dependent repression of herpes simplex virus: implications for the regulation of viral latency. Virol J 3: 44.

7. Huang X, Lu B, Yu W, Fang Q, Liu L, et al. (2009) A novel replicationcompetent vaccinia vector MVTT is superior to MVA for inducing high levels of neutralizing antibody via mucosal vaccination. PLoS ONE 4: e4180.
8. Liu H, Yu X, Tang X, Wang H, Ouyang W, et al. (2010) The route of inoculation determines the tissue tropism of modified vaccinia Tiantan expressing the spike glycoprotein of SARS-CoV in mice. J Med Virol 82: 727-734.

9. Voellmy R, Bloom DC, Vilaboa N (2015) A novel approach for addressing diseases not yielding to effective vaccination? Immunization by replication-competent controlled virus. Expert Rev. Vaccines 14: 637-651.

10. Voellmy R, Ahmed A, Schiller P, Bromley P, Rungger D (1985) Isolation and functional analysis of a human 70'000 dalton heat shock protein gene segment. Proc Natl Acad Sci USA 82: 4949-4953.

11. Dreano M, Brochot J, Meyers A, Cheng-Meyer C, Rungger D, et al. (1986) High-level, heat-regulated synthesis of proteins in eukaryotic cells. Gene 49: 1-8.

12. Brade AM, Ngo D, Szmitko P, Li P-X, Liu F-F, et al. (2000) Heat-directed gene targeting of adenoviral vectors in tumor cells. Cancer Gene Ther 7: 1566-1574.

13. Vilaboa N, Fenna M, Munson J, Roberts SM, Voellmy R (2005) Novel gene switches for targeted and timed expression of proteins of interest. Mol Ther 12: 290-298.

14. Martin-Saavedra FM, Bore A, Voellmy R, Vilaboa N (2009) Heatactivated, rapamycin-dependent gene switches for tight control of transgene expression. Hum Gene Ther 20: 1060-1061.

15. Martín-Saavedra FM, Wilson CG, Voellmy R, Vilaboa N, Franceschi RT (2013) Spatiotemporal control of vascular endothelial growth factor expression using a heat shock and rapamycin-dependent gene switch. Hum Gene Ther Methods 24: 160-170.

16. Moss B, Smith GL, Gerin JL, Purcell RH (1984) Live recombinant vaccinia virus protects chimpanzees against hepatitis B. Nature 311: 67-69.

17. Draper SJ, Heeney JL (2010) Viruses as vaccine vectors for infectious diseases and cancer. Nat Rev Microbiology 8: 62-73. 